\title{
A Multicenter Study of the Prevalence of Dry Eye Disease in Chinese Refractive Surgery Candidates
}

\author{
Meiyan Li ${ }^{a, b, c} \quad$ Li Zeng $^{a, b, c}$ Shengjian Mid ${ }^{\text {Ying Lie }}$ Zhirong Liuf \\ Keming Yu ${ }^{g}$ Qi Hu ${ }^{\mathrm{h}}$ Haiyan $\mathrm{Li}^{\mathrm{i}}$ Daijin Maj Yuehua Zhou ${ }^{\mathrm{k}}$ Jibing Wangl

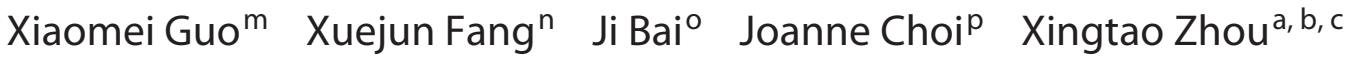 \\ aDepartment of Ophthalmology and Optometry, EENT Hospital, Fudan University, Shanghai, China; ${ }^{\mathrm{b}} \mathrm{NHC}$ Key \\ Laboratory of Myopia (Fudan University), Shanghai, China; 'Shanghai Research Center of Ophthalmology and \\ Optometry, Shanghai, China; ${ }^{d}$ Shanxi Center Hospital The Chinese Army Police Force, Taiyuan, China; ${ }^{\text {Peking }}$

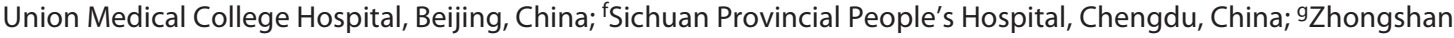

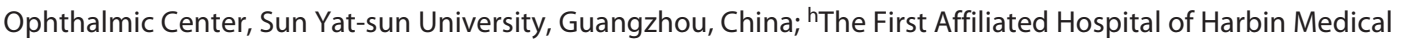 \\ University, Harbin, China; 'Shanghai Xinshijie Eye Hospital, Shanghai, China; 'Aier Eye Hospital (Changsha), \\ Changsha, China; ${ }^{B}$ Beijing Tongren Hospital, Capital Medical University, Beijing, China; 'Weifang Eye Hospital, \\ Weifang, China; 'mLixiang Eye Hospital of Soochow University, Suzhou, China; ${ }^{\mathrm{n} A i e r}$ Eye Hospital (Shenyang), \\ Shenyang, China; ${ }^{\circ}$ Chongqing Daping Hospital, Chongqing, China; ${ }^{P}$ Wayne State University School of Medicine, \\ Detroit, MI, USA
}

\section{Keywords}

Dry eye $\cdot$ Contact lens $\cdot$ Tear film breakup time $\cdot$ Schirmer test $\cdot$ Keratorefractive surgery

\begin{abstract}
Objective: The aim of this study was to determine the prevalence of preoperative dry eye disease and evaluate tear film function in refractive surgery candidates in China. Methods: In this prospective noninterventional cross-sectional study, refractive surgical candidates from 13 preselected eye hospitals in China were recruited from July 2015 to February 2016. Patient histories, subjective symptoms, tear film breakup time (TBUT), ocular surface fluorescein staining, and Schirmer I tests (SIT), were assessed to conduct subgroup analysis. Results: A total of 1,849 patients were recruited, $41.4 \%$ were diagnosed with dry eye disease $(766 / 1,849)$ and $44.9 \%(830 / 1,849)$ of subjects had a positive history of con-
\end{abstract}

karger@karger.com

(c) 2020 S. Karger AG, Basel

www.karger.com/ore

Karger! tact lens (CL) wear. The overall mean TBUT and SIT values were $7.3 \pm 3.7 \mathrm{~s}$ and $15.2 \pm 8.8 \mathrm{~mm}$, respectively. The total prevalence of ocular surface fluorescein staining was $23.46 \%$ $(422 / 1,849) ; 44.62 \%$ of patients had TBUT $<5$ s and $23.20 \%$ of patients had SIT $<5 \mathrm{~mm}$. CL wearers were observed to have a higher prevalence of dry eye than non-CL wearers (54.1 vs. $35.2 \%, \mathrm{OR}=2.17,95 \% \mathrm{Cl}: 1.77-2.65)$. Conclusions: In this study, the most common abnormal finding in dry eye disease was tear film instability. A high proportion of refractive surgery candidates have preexisting dry eye disease and a history of CL wear prior to surgery. Careful attention should be given to the evaluation of preoperative dry eye in refractive surgery candidates.

(c) 2020 S. Karger AG, Base

Meiyan Li and Li Zeng contributed equally to this work and are cofirst authors.
Xingtao Zhou, Department of Ophthalmology and Optometry

Myopia Key Laboratory of the Health Ministry, Shanghai Research Center of Ophthalmology and Optometry, Eye and ENT Hospital of Fudan University No. 83 Fenyang Road, Shanghai 200031 (China)

doctzhouxingtao@163.com 


\section{Introduction}

Dry eye is recognized as a growing public health concern with a prevalence of $30.05 \%$ in people over the age of 20 in Shanghai [1]. It is characterized by foreign body sensation, burning or stinging, blurred vision, and photophobia [2,3]. Dry eye is a common complaint among patients who have undergone refractive surgeries, including photorefractive keratectomy, laser epithelial keratomileusis, laser in situ keratomileusis (LASIK), and small incision lenticule extraction [4-6].

It has been reported that preoperative tear volume may affect recovery of the ocular surface after corneal refractive surgery and may increase the risk for chronic dry eye [7]. Patients who develop dry eye after refractive surgery are at an elevated risk for developing subsequent refractive regression [8] and ocular surface damage [8]. Thus, it is important for corneal refractive surgeons to evaluate patients for dry eye disease before surgery in order to offer more careful perioperative management. To the best of our knowledge, this is the first study to investigate the prevalence of dry eye in patients who are candidates for refractive surgery in China, as well as identify the effect of contact lens (CL) wear on dry eye disease.

\section{Materials and Methods}

\section{Subjects}

Patients enrolled in this study were candidates for refractive surgery who had received preoperative evaluations in one of the 13 hospitals or eye centers from July 2015 to February 2016 in China. The Ethical Committee of the Fudan University Eye and ENT Hospital Review Board approved the study protocol, and the study was conducted in accordance with the principles of the Declaration of Helsinki regarding research involving human subjects. Written informed consent was obtained from all patients after a complete description of the study.

The inclusion criteria were as follows: age 18-45 years, all genders, and no restrictions on the type and degree of myopia. Subjects with any of the following conditions were excluded from this study: (1) uncontrolled systemic diseases or disabilities affecting his or her daily activities (including ocular allergies, infection not related to dry eye syndrome [DES]), (2) external ocular disease, (3) history of ocular surgery within the last 6 months, and (4) known allergy to any component of the agents used in the study (e.g., fluorescein). None of the subjects enrolled were using tear supplements on the day that they participated in the study.

Questionnaire and Dry Eye Evaluation

CL Wear History

The CL history was considered positive if the patient had worn $\mathrm{CL}>4$ times per week for $>1$ month in the past 6 months.

Prevalence of Dry Eye in Chinese

Refractive Surgery Candidates
Subjective Symptoms Inquiry

All patients were questioned with a dry eye symptom questionnaire. Patients were asked if they suffered from a frequent or sustained occurrence of any one of the following symptoms in the last month: dryness, stinging sensation, burning sensation, eye strain or fatigue, discomfort, and/or vision fluctuations.

Tear Film Breakup Time (TBUT) and Corneal Fluorescein

Staining

The ocular surface was stained using a fluorescein strip (Jingming, Tianjing, China) wet with non-preservative saline solution applied to the lower conjunctival sac. The patient was asked to blink several times. Using slit-lamp biomicroscopy with a cobalt blue filter, the tear film was observed. The time that elapsed between the last complete blink and the first observation of tear film breakup was recorded as the TBUT. The test was repeated 3 times, and the average of the 3 measurements was calculated. After the TBUT test, each cornea was examined for superficial fluorescein staining.

Schirmer's Test (without Surface Anesthesia)

The Schirmer test without anesthesia (Schirmer I test, SIT) was performed to measure tear production. A 30-mm Schirmer tear test strip (Jingming, Tianjing, China) was placed in the inferior fornix at the junction of the middle and lateral thirds of the lower eyelid margin. Schirmer test strips remained in place for $5 \mathrm{~min}$ with the eyes closed. The extent of wetting was subsequently measured according to the scale provided by the manufacturer. Potential scores ranged from 0 to $30 \mathrm{~mm}$, with lower scores indicating more severe deficiencies in tear production [9].

Dry Eye Diagnosis Standard

The dry eye diagnosis standard proposed in 2013 by the Cornea Group of Ophthalmology Society of the Chinese Medical Association was adopted in the present study. Patients were diagnosed with dry eye if: (1) patients reported subjective symptoms and either TBUT $\leq 5 \mathrm{~s}$, or SIT $\leq 5 \mathrm{~mm} / 5$ mins; (2) patients reported subjective symptoms and had positive ocular surface staining and either $5 \mathrm{~s}<$ TBUT $\leq 10 \mathrm{~s}$, or SIT $\leq 10 \mathrm{~mm} / 5 \mathrm{mins}$. Only one eye needed to meet the above criteria for a patient to be diagnosed with dry eye disease.

\section{Statistical Analysis}

According to the results of the Hong Kong study [10], 15.6\% of the patients had subjective dry eye symptoms before LASIK. A simple random sampling equation was used to calculate the sample size as $n$ (required sample size) $=Z^{2}(p)(1-p) /\left(B \times p^{2}\right), p=0.156$, $B=0.20, Z=1.96$ (when significance level was 0.05 ). As a result, $n$ equals 520. All statistical analyses were performed with SAS for Windows software (Version 9.4). The $\chi^{2}$ test or Wilcoxon rank sum tests were used to determine whether there was a significant difference in dry eye symptoms and test results between the 2 groups. A $p$ value of $<0.05$ was considered significant.

\section{Results}

Dry Eye Parameters

A total of 1,849 patients were included in this study. In total, $44.9 \%(830 / 1,849)$ of patients had a positive 
Fig. 1. Prevalence of dry eye among study sites.

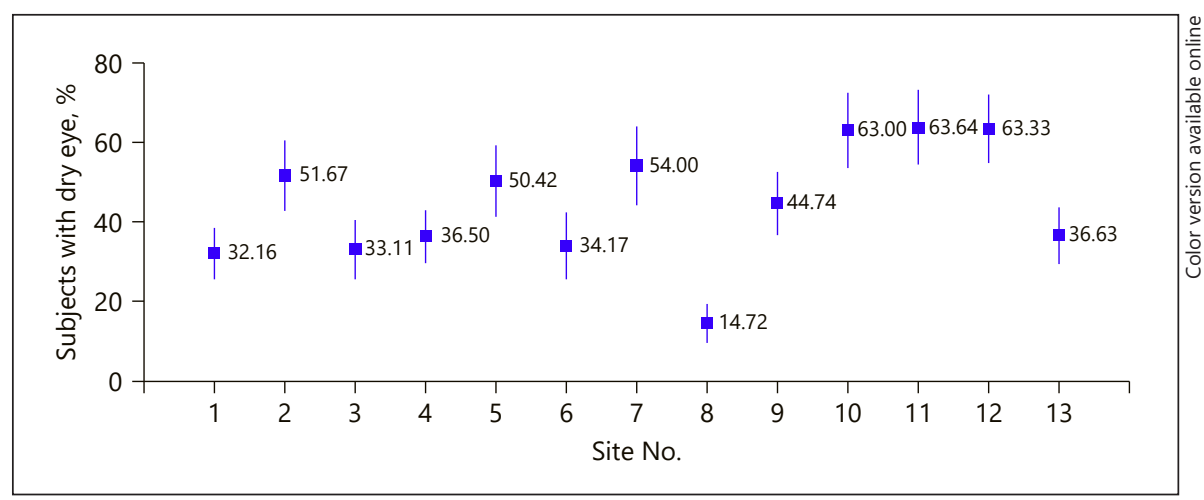

Table 1. Comparison of TBUT and SIT results

\begin{tabular}{|c|c|c|c|c|}
\hline & Overall & CL group & Non-CL group & $p$ value \\
\hline \multicolumn{5}{|l|}{ TBUT } \\
\hline $\mathrm{TBUT} \leq 5 \mathrm{~s}$ & $44.62 \%(826)$ & $52.34 \%(436)$ & $43.82 \%(337)$ & \multirow[t]{3}{*}{0.001} \\
\hline $5 \mathrm{~s}<\mathrm{BUT} \leq 10 \mathrm{~s}$ & $43.17 \%(799)$ & $38.42 \%(320)$ & $42.91 \%(330)$ & \\
\hline $\mathrm{TBUT}>10 \mathrm{~s}$ & $12.21 \%(226)$ & $9.24 \%(77)$ & $13.26 \%(102)$ & \\
\hline \multicolumn{5}{|l|}{ SIT } \\
\hline $\mathrm{SIT} \leq 5 \mathrm{~mm}$ & $23.20 \%(428)$ & $25.67 \%(212)$ & $22.82 \%(175)$ & \multirow[t]{3}{*}{0.14} \\
\hline $5 \mathrm{~mm}<\mathrm{SIT} \leq 10 \mathrm{~mm}$ & $25.53 \%(471)$ & $27 \%(223)$ & $24.9 \%(191)$ & \\
\hline $\mathrm{SIT}>10 \mathrm{~mm}$ & $51.27 \%(946)$ & $47.34 \%(391)$ & $52.28 \%(401)$ & \\
\hline \multicolumn{5}{|c|}{ Corneal fluorescein staining } \\
\hline Positive & $23.46 \%(422)$ & $29.54 \%(239)$ & $19.44 \%(146)$ & \multirow[t]{2}{*}{$<0.0001$} \\
\hline Negative & $76.54 \%(1,377)$ & $70.46 \%(570)$ & $80.56 \%(605)$ & \\
\hline
\end{tabular}

CL, contact lens; TBUT, tear film breakup time; SIT, Schirmer I test.

history of CL wear. The mean TBUT in the study was $7.3 \pm 3.7 \mathrm{~s}$. TBUT values in non-CL wearers was greater than in CL wearers $(7.4 \pm 3.8$ vs. $6.8 \pm 3.4 \mathrm{~s}, p=$ $0.002)$. The mean SIT was $15.2 \pm 8.8 \mathrm{~mm}$. SIT values in non-CL wearers were greater than in CL wearers (15.3 \pm 8.8 vs. $14.6 \pm 8.9 \mathrm{~mm}, p=0.12)$. The rate of ocular surface fluorescein staining was $22.8 \%(422 / 1,849)$. Non-CL wearers had a lower rate of ocular surface staining than compared to CL wearers ( 19.0 vs. $28.8 \%$, $p<0.0001$ ).

Further analysis revealed that CL wearers had more tear film instability with TBUT values $<5 \mathrm{~s}$, whereas nonCL wearers more frequently had TBUT values $>10 \mathrm{~s}$. Additionally, patients in the CL group had decreased tear production with Schirmer I values of $<10 \mathrm{~mm}$, whereas values $>10 \mathrm{~mm}$ were more frequently found in the nonCL group (Table 1).

\section{Prevalence of Dry Eye}

The overall prevalence of dry eye in this study was $41.43 \%(766 / 1,849)$, ranging from 14.72 to $63.64 \%$ (Fig. 1 ). CL wearers were observed to have a higher prevalence of dry eye disease than non-CL wearers ( 54.1 vs. $35.2 \%$; $\mathrm{OR}=2.17,95 \% \mathrm{CI}: 1.77-2.65)$. Stratified results analysis showed similar trends across most sites (Fig. 2).

Table 2 presents the prevalence of dry eye with different diagnostic criteria. For the overall patients, the most occurring dry eye diagnosis outcome was subjective symptoms combined with TBUT $\leq 5 \mathrm{~s}$, followed by subjective symptoms combined with SIT $\leq 5 \mathrm{~mm}$.

\section{Discussion}

The health of the ocular surface is important for corneal refractive surgery. Tear film abnormalities associated with dry eye disease not only affects corneal topogra- 
Fig. 2. Forest plots for comparison between CL wearers and non-CL wearers in prevalence of dry eye among study sites. CL, contact lens.

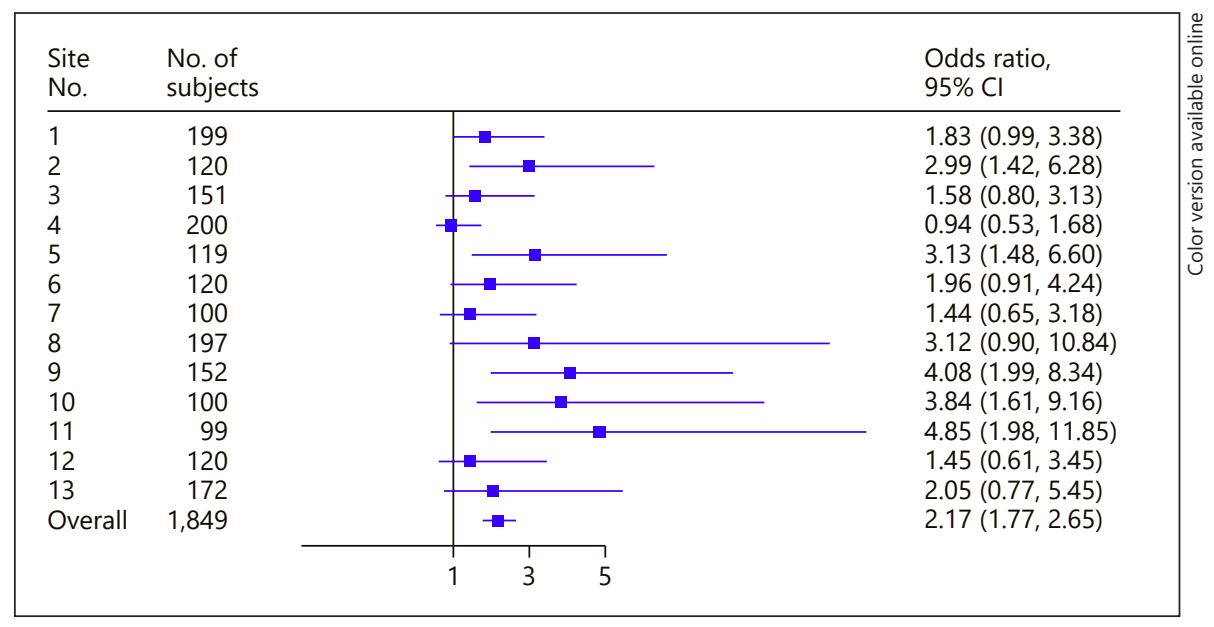

Table 2. Incidence of dry eye $(n / \%)$

\begin{tabular}{|c|c|c|c|c|}
\hline \multicolumn{5}{|l|}{ Dry eye diagnostic criteria } \\
\hline (1) Symptoms of dry eye + BUT $\leq 5 \mathrm{~s}$ & $31.17 \%(582)$ & $40.96 \%(342)$ & $26.98 \%(208)$ & $<0.0001$ \\
\hline (3) Symptoms of dry eye $+5 \mathrm{~s}<\mathrm{BUT} \leq 10 \mathrm{~s}+$ positive corneal fluorescein staining & $7.23 \%(135)$ & $9.7 \%(81)$ & $5.45 \%(42)$ & 0.0014 \\
\hline (4) Symptoms of dry eye $+5 \mathrm{~mm}<$ SIT $\leq 10 \mathrm{~mm}+$ positive corneal fluorescein staining & $5.95 \%(111)$ & $8.62 \%(72)$ & $4.28 \%(33)$ & 0.0004 \\
\hline Dry eye subjective symptoms (persons, $n$ ) & $56.51 \%(1,055)$ & $68.86 \%(575)$ & $49.03 \%(378)$ & $<0.0001$ \\
\hline
\end{tabular}

CL, contact lens; SIT, Schirmer I test.

phy, including corneal anterior elevation and pachymetry $[11,12]$, but also affects recovery of the ocular surface after LASIK [13-15]. Therefore, it is important to identify dry eye disease during the preoperative evaluation. This large-scale multi-center cross-sectional study focused on prevalence of dry eye disease and dry eye parameters in a population of corneal refractive surgery candidates in China.

Several studies have investigated the prevalence of dry eye disease in different corneal refractive surgery populations. A study conducted in Hong Kong reported that $15.6 \%(15 / 96)$ of patients who underwent LASIK had dry eye symptoms preoperatively [16]. Additionally, another study performed in Australia showed that $8 \%(n=45 / 565)$ of LASIK candidates had dry eye disease [8]. The prevalence of dry eye disease in Russian patients $(n=400)$ with myopia being evaluated for LASIK was estimated at approximately $10-40 \%$ (based on clinical signs) and $40-$ $55 \%$ (based on symptoms) [17]. In the present study, the overall prevalence of dry eye in this study was $41.43 \%$

Prevalence of Dry Eye in Chinese

Refractive Surgery Candidates
$(766 / 1,849)$. Of note, the prevalence of dry eye among different study sites was variable and ranged from 14.72 to $63.64 \%$. Dry eye is a multifactorial disease, thus cultural, geographic, climatic, genetic, or socio-economic differences between different regions may contribute to the difference in prevalence between our 13 sites.

In addition, different diagnostic criteria for dry eye disease were used in the aforementioned studies which may be a factor in the discrepancies between results. Therefore, we have also included the objective measures of dry eye parameters. A study conducted in an Iranian population of 655 patients showed that mean TBUT and Schirmer tests with anesthesia were $14.3 \mathrm{~s}$ and $15.5 \mathrm{~mm}$, respectively. TBUT and Schirmer tests with anesthesia were abnormal in $30.8 \%$ (TBUT $\leq 10 \mathrm{~s}$ ) and $14.6 \%$ (SIT $\leq 10 \mathrm{~mm}$ ) of cases in patients undergoing screening for myopic laser keratorefractive surgery [18]. In Russian patients $(n=400)$, the mean Schirmer test score was 15.2 $\mathrm{mm}$ and $36.5 \%$ of patients had evidence of tear volume deficiency (Schirmer score $\leq 10 \mathrm{~mm}$ ). The mean TBUT 
was $11.7 \mathrm{~s}$ and $10.1 \%$ of patients had tear film instability (TBUT $\leq 5 \mathrm{~s})$ [17]. In our present study, the mean Schirmer score was $15.2 \mathrm{~mm}$ and $48.73 \%$ of patients had tear production deficiency (Schirmer score $\leq 10 \mathrm{~mm}$ ). The mean TBUT was $7.3 \mathrm{~s}$ and $44.62 \%$ of patients had tear film instability (TBUT $\leq 5 \mathrm{~s}$ ). Even when patients with CL wear history were excluded, the mean TBUT value was $7.4 \mathrm{~s}$, and $43.82 \%$ of patients had a TBUT $<5 \mathrm{~s}$. This is significantly shorter than what has previously been reported. In addition, the most commonly met diagnostic criteria for dry eye disease were subjective symptoms combined with TBUT $\leq 5$. These results indicate that the most common dry eye test abnormity is tear film instability in Chinese refractive surgery candidates.

CL usage can lead to changes in the ocular surface. In vivo confocal microscopy showed significantly decreased basal epithelial cell density, lower acinar unit diameters, higher glandular orifice diameters, greater secretion reflectivity, and greater homogeneity of the periglandular interstices in CL wearers compared to controls, indicating meibomian gland dropout, duct obstruction, and glandular inflammation [19]. Literature has shown that CL wear was also related to changes in ocular surface and tear volume parameters [20-22]. Yang et al. [23] reported that CL wear (OR, 2.4; 95\% CI, 1.3-4.4) was a risk factor for developing dry eye. According to data offered by Pili et al. [20], approximately $38.1 \%$ of Caucasian soft CL and rigid gas-permeable lens wearers had both OSDI and TBUT abnormalities, and the rate of reported OSDI abnormality was $64.3 \%$. Similarly, in our present study, the prevalence of dry eye disease was significantly higher in CL wearers than non-CL wearers in all sites. In the clinic setting, complaints of dry eye symptoms could be a motivating factor for CL-wearing patients to undergo refractive surgeries [24]; therefore, more attention should be paid to CL wearers.

Although preoperative dry eye is a major risk factor for developing severe dry eye after surgery and should be identified prior to surgery, optimization of the ocular surface can be achieved prior to surgery using artificial tears, nutritional supplements, punctal occlusion, and topical cyclosporine A. This can decrease the incidence of worsening dry eye symptoms following surgery [25].

There are several limitations to this study. First, this study did not analyze the effect of CL type on ocular surface health. Second, this multicenter study involved different regions of China, namely, different humidity and temperature, which may cause a bias to the dry eye examination results; however, stratified results analysis showed similar trends across most sites.
In conclusion, dry eye disease and subjective symptoms of dry eye rates were higher in preoperative keratorefractive surgery candidates, especially those who had been CL wearers before intervention. Preoperative dry eye evaluation and treatments are recommended particularly for CL wearing patients who are considering keratorefractive surgery.

\section{Acknowledgements}

The authors thank the research staff members from the 13 hospitals for their contribution to this article.

\section{Statement of Ethics}

The study was approved by the ethics committee of the Eye and ENT Hospital of Fudan University and was conducted ethically in accordance with the World Medical Association Declaration of Helsinki. Subjects have given their written informed consent and that the study protocol was approved by the institute's committee on human research.

\section{Conflict of Interest Statement}

The authors have no conflicts of interest to disclose.

\section{Funding Sources}

This study was supported by the National Natural Science Foundation of China (Grant No. 81500753; Grant No. 81770955) and Project of Shanghai Science and Technology (Grant No. 17140902900; Grant No. 17411950200).

\section{Author Contributions}

M.L., L.Z., S.M., Y.L., Z.L., K.Y., Q.H., H.L., D.M., Y.Z., J.W., X.G., X.F., and J.B. acquired the data. M.L. and L.Z. analyzed the patient data and drafted the manuscript. J.C. revised the manuscript. X.Z. conducted the surgery and revised the manuscript critically. All authors have read and approved the final manuscript.

\section{Availability of Data and Material}

The datasets used and/or analyzed during the current study are available from the corresponding author on reasonable request.

\section{Consent for Publication}

Informed consent was obtained from all participants. 


\section{References}

1 Tian YJ, Liu Y, Zou HD, Jiang YJ, Liang XQ, Sheng MJ, et al. [Epidemiologic study of dry eye in populations equal or over 20 years old in Jiangning District of Shanghai]. Zhonghua Yan Ke Za Zhi. 2009;45(6):486-91.

2 Begley CG, Caffery B, Chalmers RL, Mitchell GL. Use of the dry eye questionnaire to measure symptoms of ocular irritation in patients with aqueous tear deficient dry eye. Cornea. 2002;21(7):664-70.

3 Li M, Gong L, Chapin WJ, Zhu M. Assessment of vision-related quality of life in dry eye patients. Invest Ophthalmol Vis Sci. 2012; 53(9):5722-7.

4 Mian SI, Li AY, Dutta S, Musch DC, Shtein RM. Dry eyes and corneal sensation after laser in situ keratomileusis with femtosecond laser flap creation effect of hinge position, hinge angle, and flap thickness. J Cataract Refract Surg. 2009;35(12):2092-8.

5 Salomao MQ, Ambrosio RJ, Wilson SE. Dry eye associated with laser in situ keratomileusis: mechanical microkeratome versus femtosecond laser. J Cataract Refract Surg. 2009; 35(10):1756-60.

6 Hammond MD, Madigan WP, Bower KS. Refractive surgery in the United States Army, 2000-2003. Ophthalmology. 2005; 112(2): $184-90$.

7 Konomi K, Chen LL, Tarko RS, Scally A, Schaumberg DA, Azar D, et al. Preoperative characteristics and a potential mechanism of chronic dry eye after LASIK. Invest Ophthalmol Vis Sci. 2008;49(1):168-74.

8 Albietz JM, Lenton LM, McLennan SG. Chronic dry eye and regression after laser in situ keratomileusis for myopia. J Cataract Refract Surg. 2004;30(3):675-84.
9 Vitale S, Goodman LA, Reed GF, Smith JA. Comparison of the NEI-VFQ and OSDI questionnaires in patients with Sjögren's syndrome-related dry eye. Health Qual Life Outcomes. 2004;2:44.

10 Liu NN, Liu L, Li J, Sun YZ. Prevalence of and risk factors for dry eye symptom in mainland china: a systematic review and meta-analysis. J Ophthalmol. 2014;2014:748654.

11 Asena L, Altınörs DD, Cezairlioğlu Ş, Bölük SO. Effect of dry eye on Scheimpflug imaging of the cornea and elevation data. Can J Ophthalmol. 2017;52(3):313-7.

12 Lee JH, Kim JH, Kim SW. Repeatability of central corneal thickness measurement using rotating Scheimpflug camera in dry and normal eyes. Eye Contact Lens. 2018;44(Suppl 2): S29-32.

13 Konomi K, Chen LL, Tarko RS, Scally A, Schaumberg DA, Azar D, et al. Preoperative characteristics and a potential mechanism of chronic dry eye after LASIK. Invest Ophthalmol Vis Sci. 2008;49(1):168-74

14 Ambrosio RJ, Wilson SE. Complications of laser in situ keratomileusis: etiology, prevention, and treatment. J Refract Surg. 2001; 17(3):350-79.

15 Liang L, Zhang M, Zou W, Liu Z. Aggravated dry eye after laser in situ keratomileusis in patients with Sjögren syndrome. Cornea. 2008; 27(1):120-3.

16 Yu EY, Leung A, Rao S, Lam DS. Effect of laser in situ keratomileusis on tear stability. Ophthalmology. 2000;107(12):2131-5.

17 Maychuk DY. Prevalence and severity of dry eye in candidates for laser in situ keratomileusis for myopia in Russia. J Cataract Refract Surg. 2016;42(3):427-34.
18 Farahi A, Hashemi H, Mehravaran S, Tavakolizadeh S, Khabazkhoob M. Tear function evaluation in candidates of corneal laser refractive surgery for myopia. Eye Contact Lens. 2014;40(2):91-4.

19 Villani E, Ceresara G, Beretta S, Magnani F, Viola F, Ratiglia R. In vivo confocal microscopy of meibomian glands in contact lens wearers. Invest Ophthalmol Vis Sci. 2011; 52(8):5215-9.

20 Pili K, Kaštelan S, Karabatić M, Kasun B, Čulig B. Dry eye in contact lens wearers as a growing public health problem. Psychiatr Danub. 2014;26(Suppl 3):528-32.

21 Vajdic C, Holden BA, Sweeney DF, Cornish RM. The frequency of ocular symptoms during spectacle and daily soft and rigid contact lens wear. Optom Vis Sci. 1999;76(10):70511.

22 Guillon M, Dumbleton K, Theodoratos P, Patel T, Karkkainen T, Moody K. Objective assessment of ocular surface response to contact lens wear in presbyopic contact lens wearers of Asian descent. Eye Contact Lens. 2018; 44(3): 182-9.

23 Yang WJ, Yang YN, Cao J, Man ZH, Yuan J, Xiao X, et al. Risk factors for dry eye syndrome: a retrospective case-control study. Optom Vis Sci. 2015;92(9):e199-205.

24 Shtein RM. Post-LASIK dry eye. Expert Rev Ophthalmol. 2011;6(5):575-82.

25 Ambrosio RJ, Tervo T, Wilson SE. LASIK-associated dry eye and neurotrophic epitheliopathy: pathophysiology and strategies for prevention and treatment. J Refract Surg. 2008;24(4):396-407. 